\title{
ArcheoSciences
}

Revue d'archéométrie

$30 \mid 2006$

Varia

\section{La grotte de Kulna : analyses physico-chimique et radiométrique des os et dentines de grands mammifères des niveaux du Paléolithique moyen}

Kulna Cave : physicochemical and radiometric analyses of large mammal bones and dentine from Middle Paleolithic levels

Véronique Michel, Hervé Bocherens, Karel Valoch et Yuji Yokoyama

\section{OpenEdition}

Journals

Édition électronique

URL : https://journals.openedition.org/archeosciences/294

DOI : 10.4000/archeosciences.294

ISBN : 978-2-7535-1595-6

ISSN : 2104-3728

Éditeur

Presses universitaires de Rennes

Édition imprimée

Date de publication : 31 décembre 2006

Pagination : 137-142

ISBN : 978-2-7535-0456-1

ISSN : 1960-1360

Référence électronique

Véronique Michel, Hervé Bocherens, Karel Valoch et Yuji Yokoyama, «La grotte de Kulna : analyses physico-chimique et radiométrique des os et dentines de grands mammifères des niveaux du Paléolithique moyen », ArcheoSciences [En ligne], 30 | 2006, mis en ligne le 31 décembre 2008, consulté le 01 février 2022. URL : http://journals.openedition.org/archeosciences/294 ; DOI : https:// doi.org/10.4000/archeosciences.294 


\title{
La grotte de Kůlna : analyses physico-chimique et radiométrique des os et dentines de grands mammifères des niveaux du Paléolithique moyen
}

\author{
Véronique MicheL*, Hervé Bocherens**, Karel Valoch*** et Yuji Yokoyama****
}

\begin{abstract}
Résumé : Le site de Kủlna est situé en Moravie au nord de Brno (République tchèque). Des fouilles conduites par Valoch, de 1961 à 1976 , ont livré des dépôts du Paléolithique supérieur et moyen. L'examen visuel de l'ensemble des restes fauniques récoltés dans les niveaux du Paléolithique moyen (industrie micoquienne et taubachienne) et conservés au Musée Moravské zemské à Brno, montre une large variété de couleur. Treize échantillons de faune plus ou moins colorés ont été analysés par différentes méthodes physico-chimiques et par spectrométrie alpha pour une tentative de datation U-Th. La matière organique et la phase minérale apatite des restes fauniques sont bien conservées. La coloration des échantillons est liée à la présence d'oxydes de manganèse et de fer contaminants. Les âges U-Th déterminés en supposant un système fermé sont dispersés, ce qui ne permet pas d'apporter un résultat chronologique convenable en comparaison avec les résultats ESR et C-14 publiés par ailleurs.
\end{abstract}

Abstract: The Külna site is located in Moravia about $30 \mathrm{~km}$ North of Brno (Czech Republic). Excavations conducted by Valoch between 1961-1976, revealed Middle and Upper Paleolithic levels. A visual examination of the faunal remains collected from the Middle Paleolithic (micoquian and taubachian industries) and preserved at the Moravské zemské Museum, in Brno, display a wide range of colours. Thirteen samples of faunal remains with different colours, were analysed by using several physicochemical methods and by alpha spectrometry for a U-Th dating assessment. The organic matter and the mineral part of the faunal remains are well preserved. The colouring of the samples is due to the presence of manganese and iron oxides. The $U$ Th ages calculated by assuming a closed system are dispersed, that yield not to a credible chronological result in comparison to the ESR and C-14 results published by other authors.

Mots clés : Os, état de conservation, collagène, coloration, cristallinité, U/Th, datation, Paléolithique moyen.

Key words: Bone, preservation state, collagen, colouring, crystallinity, U/Th, dating, Middle Paleolithic.

\section{INTRODUCTION}

La grotte de Kůlna se trouve à environ $30 \mathrm{~km}$ au nord de Brno et à une altitude de $470 \mathrm{~m}$ (Fig. 1). C'est une vaste cavité creusée dans le Karst morave, en forme de tunnel long de $87 \mathrm{~m}$, large de $25 \mathrm{~m}$ au maximum et haute d'environ
$8 \mathrm{~m}$. Après des fouilles organisées pendant les années 19611976, le site a fait l'objet de plusieurs études (Valoch, 1988; Rink et al., 1996). Le remplissage atteint $15 \mathrm{~m}$ d'épaisseur et 14 niveaux ont été décrits par Valoch (Fig. 2). La séquence stratigraphique contient des dépôts du Paléolithique moyen et supérieur. Notre étude concerne les niveaux médians (11

\footnotetext{
* Géosciences Azur, Université de Nice-Sophia Antipolis, Parc Valrose, 06108 Nice cedex 02, France; Cépam, UMR 6130, 250 rue Albert-Einstein, 06560 Valbonne, France. (vmichel@cepam.cnrs.fr)

** Institut des Sciences de l'Évolution, UMR 5554, Université Montpellier 2, Place Eugène-Bataillon, 34090 Montpellier cedex 05, France. (bocheren@ isem.univ.montp2.fr)

*** Moravské zemské museum, CZ-65937 Brno, Czech Republic. (kvaloch@mzm.cz)

*** Institut de paléontologie humaine, MNHN, 1 rue René-Panhard, 75013 Paris.
} 


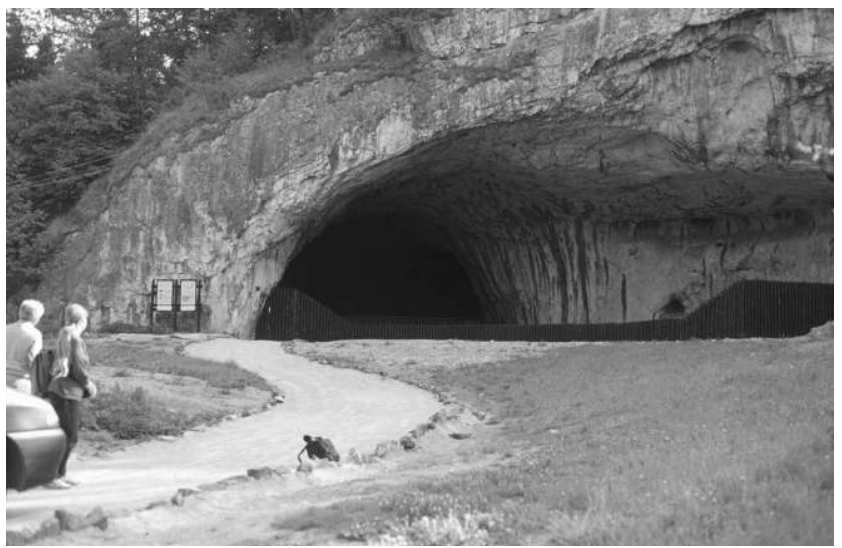

Figure 1 : L’entrée de la Grotte de Kůlna.

Figure 1: Külna Cave entrance.

à 6a) correspondant au Paléolithique moyen (14 à 6a) avec la présence d'une industrie micoquienne et taubachienne (Fig. 2). Dans le niveau micoquien $7 \mathrm{a}$, des restes humains de Néandertal (deux os du crâne et trois dents de lait) ont été découverts (Jelinek, 1988). D'après Condemi (in PatouMathis et al., 2005) ceux-ci appartiennent à la dernière phase de l'évolution des néandertaliens en Europe. Des traces de feu ont été découvertes dans le niveau $7 \mathrm{a}$ et quelques lentilles de charbon dans le niveau 11. Les résultats des analyses UTh sont présentés et comparés aux datations publiées par Mook (1988) et Rink et al. (1996). Pour tenter d'évaluer l'état de conservation et d'expliquer la coloration des fossiles des analyses physico-chimiques ont été conduites. Les résultats détaillés sont présentés par ailleurs (Michel et al., 2006). Ils sont parallèlement résumés dans le cadre de ce travail. En effet, pour la datation U-Th, il est important de connaitre l'état de conservation des os. D'une manière générale, Un os brûlé a subi une recristallisation de la phase minérale apatite. Il contiendrait moins d'uranium à l'état fossile qu'un os non brûlé fossile issu du même environnement (Michel et al., 1997). D'autre part, la coloration de l'os par le manganèse ou le fer, témoigne de changements redox conduisant à des migrations de l'uranium, soit à une ouverture du système.

\section{RÉCAPITULATIF DES DATATIONS Des niveaux du Paléolithique Moven}

En 1996, 28 échantillons d'émail dentaire de grands mammiferes tels que Equus sp., Rangifer tarandus, Bos primigenius, Cervus elaphus et Mammuthus primigenius ont été analysés par ESR (Fig. 2) et deux dents par U-Th par spectrométrie de masse à ionisation thermique (Rink et al., 1996). Globalement, en datation ESR, il n'apparaît pas de cohérence des âges avec la séquence stratigraphique du fait des moyennes d'âges jeunes obtenues sur des dents de grands mammiferres dans le niveau $7 \mathrm{c}$ et dans les niveaux 11a-11c. En écartant les âges anormalement jeunes, les auteurs concluent à une moyenne d'âges ESR (EU: early uptake - LU: linear uptake) comprise entre 46 et 50 ka pour le niveau 7 a, ce qui est en accord avec la datation ${ }^{14} \mathrm{C}$ non calibrée obtenue par la méthode ${ }^{14} \mathrm{C}$ classique (Mook, 1988) (Fig. 2). L'auteur publie trois dates ${ }^{14} \mathrm{C}$ différentes pour les mêmes niveaux archéologiques. Une date de $45,6_{-2,2}^{+2,8} \mathrm{ka} \mathrm{BP}(\mathrm{GrN}-6060)$ a été obtenue par l'analyse d'un échantillonnage d'os brûlés. Une seconde analyse a été réalisée sur un extrait d'un échantillonnage d'os brûlés qui ont été traités afin d'éliminer les acides humiques. Elle conduit à une datation plus jeune de $38,6_{-0,8}^{+0,95} \mathrm{ka} \mathrm{BP}$ (GrN-6024) (Mook, 1988). Les os sont dits brûlés, peutêtre ont-ils été décrits ainsi à cause de leur couleur? En effet, la majeure partie des os de Kůlna est plus ou moins colorée (voir ci-dessous). Enfin, un charbon (GrN-10347) a été daté de 36,4 ka BP. En tenant compte du fait que les âges C-14 sont non calibrés et au vu des résultats de datation ESR, il semble correct de considérer que l'âge du niveau 7a est compris entre 46 et $50 \mathrm{ka}$ comme l'ont suggéré Rink et al. (1996). Les mêmes auteurs concluent qu'une date ESR de 69 \pm 8 ka serait correcte pour le niveau 9b (Figure 2). Les âges ESR jeunes seraient liés au fait que les niveaux sont occasionnellement riches en blocs, un facteur difficile à prendre en compte et conduisant à une surestimation de la dose annuelle (Rink et al., 1996). Les analyses U-Th (TIMS) des dents du niveau $7 \mathrm{c}$ ont donné des âges aussi vieux que 36,8 ka ou plus jeunes de 13,5 et 15,0 ka alors que la moyenne ESR correspondante est de $22 \mathrm{ka}$ (Rink et al., 1996).

\section{Matériel et MÉTHOdes}

Les échantillons ont été prélevés dans les niveaux archéologiques les plus anciens. Ces derniers sont principalement constitués de loess (niveaux 6a, 6b), d'argile (niveaux 7a, 7c) et de sable (niveau 11) (Fig. 2). L'échantillonnage a été réalisé au Musée Moravské zemské dans les collections des fouilles de 1961-1976. À partir d'une série d'une quarantaine d'échantillons fauniques destinés à une étude paléobiochimique (Bocherens, in Patou-Mathis et al., 2005), treize échantillons dont trois dents et dix fragments osseux ont été sélectionnés pour une étude sur la conservation et pour des analyses radiométriques U-Th. Les échantillons sont présentés par Michel et al. (2006) avec une description de la couleur et un code de couleur de Munsel. Les restes fauniques ont été photographiés et pour les dents des moulages ont été réalisés avant la préparation pour analyse. Les échantillons 


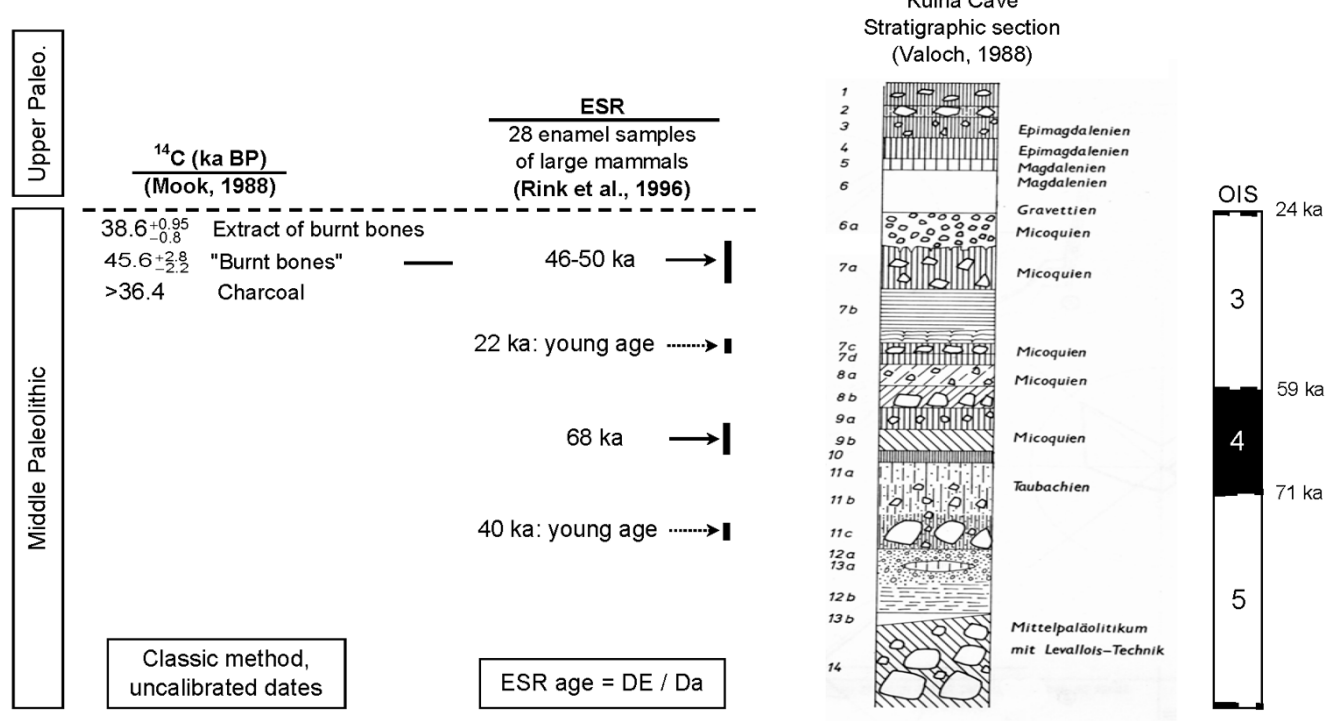

Figure 2 : Récapitulatif des datations des niveaux paléolithiques étudiés (Mook, 1988; Rink et al., 1996) et séquence stratigraphique de la grotte de Kủlna (Valoch, 1988).

Figure 2: Earlier dating data of Paleolithic levels studied (Mook, 1988; Rink et al., 1996) and stratigraphical sequence of Külna cave (Valoch, 1988).

ont été nettoyés en utilisant une fraiseuse de dentiste et mis en poudre dans un mortier en agate.

\subsection{Analyses physico-chimiques}

Les poudres d'échantillons ont été analysées pour la détermination des teneurs en Mn et Fe par spectrométrie d'absorption atomique (AA) par le laboratoire de Nancy (CRPG) afin de valider la présence éventuelle d'oxydes métalliques. Des analyses par diffraction des rayons X, par spectrométrie infrarouge à transformée de Fourier et par spectrométrie de résonance de spin électronique ESR ont été effectuées pour évaluer l'état de conservation des fossiles. Les procédures sont détaillées par ailleurs (Michel et al., 2006). Les teneurs en carbone et en azote ont été mesurées par un analyseur élémentaire CHN Carlo Erba NA 1500 en utilisant la procédure reportée dans Bocherens et al. (1997); elles sont présentées par Michel et al. (2006).

\subsection{Analyse radiométrique (méthode $\mathrm{U}-\mathrm{Th}$ )}

De 0,5 à $1,5 \mathrm{~g}$ de poudre d'ossement ou de dentine ont été prélevés pour l'analyse U-Th. L'uranium et le thorium ont été séparés en utilisant la procédure chimique de Bischoff $e t$ al. (1988) (Michel et al., 2000). Les teneurs en uranium et les rapports d'activité actuels ${ }^{234} \mathrm{U} /{ }^{238} \mathrm{U}$ et ${ }^{230} \mathrm{Th} /{ }^{234} \mathrm{U}$ ont été mesurés par spectrométrie alpha (IPH, MNHN). Les rap- ports d'activité sont reportés dans la fonction d'âge classique en utilisant un programme informatique pour obtenir l'âge par itération (Ivanovich \& Harmon, 1992). Les âges U-Th calculés sont supposés correspondre à un système fermé en considérant que les os ont subi une incorporation de tout l'uranium au début de l'enfouissement (à t=0).

\section{RÉSUlTATS, DisCUSSION}

\subsection{Analyse physico-chimique}

Le site de Kůlna a livré des os et des dents d'animaux qui présentent pour la plupart une large variété de couleur telle que le noir, le gris, le marron, le blanc, le jaune. Les teneurs en carbone et en azote mesurées ainsi que l'analyse des spectres infrarouge montrent que le collagène est plutôt bien conservé pour l'ensemble des échantillons avec des teneurs en $\mathrm{N}$ comprises entre 1,6 et 3,7 \% et en $\mathrm{C}$ comprises entre 6,8 et $12,5 \%$ à l'exception de trois os (okR9, okR7 et okR10: $\% \mathrm{~N}<1, \% \mathrm{C}<4$ ). Les spectres IR indiquent la présence d'apatite carbonatée. Le spectre de l'os spongieux okR10 montre la présence du quartz en quantité significative. Les indices de cristallinité mesurés à partir des spectres Infrarouge (SF) sont inférieurs à 3,4 et par diffraction des $\mathrm{RX}(\mathrm{CI})$ sont inférieurs à 0,1 , ce qui indique peu de changements structuraux (Michel et al., 2006). Seul, l'os okR9 pré- 
sente en spectrométrie IR un degré de cristallinité supérieur avec un indice SF de 3,5, également observé par diffraction des rayons $\mathrm{X}$ avec un indice CI de 0,15. Les analyses ESR n'indiquent pas la présence d'un radical carbone avec un signal à g = 2,004 -2005 correspondant à une chauffe de l'os à une température supérieure à $200^{\circ} \mathrm{C}$. Cependant les spectres ESR révèlent la présence de fer et de manganèse (Michel et al., 2006) . Globalement, les analyses physicochimiques montrent que les restes fauniques analysés n'ont pas été brûlés. La coloration plus ou moins foncée est liée à la présence d'oxydes de manganèse et de fer avec des teneurs en $\mathrm{Mn}$ comprises entre 0,04 et $0,57 \%$ poids et en Fe entre 0,01 et $1,61 \%$ poids (Tableau 1 ).

\subsection{Analyse radiométrique (méthode U-Th)}

Les teneurs en uranium des os et dentines sont comprises entre 0,7 et 20,7 ppm (Tableau 1 ). Elles sont très variables à l'intérieur même des différents niveaux (6a, 6b, 7a et 11). Les âges des os et des dentines sont dispersés et compris entre $10 \mathrm{ka}$ et $70 \mathrm{ka}$ (Fig. 3). Les rapports d'activité ${ }^{234} \mathrm{U} /{ }^{238} \mathrm{U}$ sont relativement élevés, supérieurs à 1,2 . Les rapports d'activité ${ }^{230} \mathrm{Th} /{ }^{232} \mathrm{Th}$ sont supérieurs à 20 , n'indiquant pas de contamination détritique de thorium. Les âges U-Th des échantillons d'os et de dentine sont représentés en fonction de leur position stratigraphique (niveaux 11, 7c, 7a, 6a-b) (Fig. 3). D'après les résultats des analyses physico-chimiques réalisées parallèlement, nous ne trouvons pas de relation significative entre la teneur en $U$ et les âges obtenus avec d'une part la couleur de l'échantillon, les degrés de cristallinité, les teneurs en matière organique et avec d'autre part les teneurs en manganèse et en fer (Tableau 1).

La surface ok100518s de l'échantillon osseux ok100518 conduit à un âge de $21 \mathrm{ka}$ avec une teneur en uranium de 17,6 ppm et une partie intérieure dont l'âge est de 42 ka avec une teneur en uranium plus faible de $0,7 \mathrm{ppm}$ (Figure 3). La totalité de l'os ok100518t conduit à une date de $20 \mathrm{ka}$ avec une teneur en uranium de $8,5 \mathrm{ppm}$. Cet os montre un profil de concentration en uranium qui a déja été observé et décrit par ailleurs (Rae \& Ivanovich, 1986; Rae et al., 1989). De tels profils indiquent une migration en uranium beaucoup plus complexe qu'une simple incorporation de tout l'uranium en début d'enfouissement (early uptake model). Afin de déterminer les âges avec plus de crédibilité, et au lieu d'utiliser le modèle du système clos (EU early uptake system) comme nous l'avons fait ici, Pike et al. (2002) suggèrent une mesure systématique des profils en uranium pour l'application de leur modèle de diffusion-adsorption (perte ou entrée de UVI). À l'exception de l'os ok100518 qui constitue une référence dans cette étude, tous les autres ossements ont été analysés en entier. Nous pouvons cependant nous appuyer sur les données ESR de Rink et al. (1996) pour comparaison. Par exemple, la partie interne ok100518i a donné l'âge U-Th le plus cohérent ( $42 \mathrm{ka}$, niveau $6 \mathrm{~b}$ ) en comparaison avec la moyenne ESR de 46-50 ka (Fig. 1). La totalité de l'os a été contaminée par de l'uranium qui diffuse de la surface vers l'intérieur de l'os, ce qui conduit à un âge plus jeune (Fig. 3). Le groupe 1 présenté en figure 3 comprend les échantillons qui ont montré le même comportement que l'os ok100518. Les âges du second groupe

\begin{tabular}{|c|c|c|c|c|c|c|c|c|}
\hline Echantillon $\mathrm{N}^{\circ}$ & Niveau & $\mathrm{Fe}^{*}(\%$ poids) & $\mathrm{Mn}^{*}(\%$ poids $)$ & $\mathrm{U}(\mathrm{ppm})$ & ${ }^{234} U /{ }^{238} U$ & ${ }^{230} \mathrm{Th} /{ }^{232} \mathrm{Th}$ & ${ }^{230} T h h^{234} U$ & Age (ka) \\
\hline ok083966d & $6 a$ & 0,05 & 0,39 & 5,0 & $1,252 \pm 0,034$ & $>1000$ & $0,254 \pm 0,009$ & $32 \pm 1$ \\
\hline ok100518 & $6 b$ & & & 8,5 & $1,268 \pm 0,027$ & $>1000$ & $0,167 \pm 0,006$ & $20 \pm 1$ \\
\hline ok100518s & & 0,02 & 0,32 & 17,6 & $1,333 \pm 0,016$ & $>1000$ & $0,179 \pm 0,004$ & $21 \pm 1$ \\
\hline ok100518i & & 0,01 & 0,01 & 0,7 & $1,261 \pm 0,110$ & $>100$ & $0,324 \pm 0,028$ & $42 \pm 5$ \\
\hline oKR11d & $6 a$ & 0,06 & 0,11 & 0,8 & $1,507 \pm 0,094$ & $>100$ & $0,438 \pm 0,028$ & $60 \pm 5$ \\
\hline oKR11d & & & & 1,3 & $1,374 \pm 0,053$ & $>1000$ & $0,430 \pm 0,018$ & $59 \pm 3$ \\
\hline okR3 & $7 a$ & 0,01 & 0,57 & 20,7 & $1,414 \pm 0,022$ & $>1000$ & $0,124 \pm 0,004$ & $14 \pm 1$ \\
\hline okR4 & $7 a$ & & & 6,1 & $1,435 \pm 0,031$ & $>1000$ & $0,490 \pm 0,014$ & $70 \pm 3$ \\
\hline ok101396 & $7 \mathrm{C}$ & 0,06 & 0,35 & 2,1 & $1,308 \pm 0,045$ & $>1000$ & $0,351 \pm 0,014$ & $46 \pm 2$ \\
\hline ok101433 & $7 c$ & 0,85 & 0,41 & 15,4 & $1,373 \pm 0,025$ & $>100$ & $0,099 \pm 0,004$ & $11 \pm 1$ \\
\hline okR5 & $7 \mathrm{c}$ & 0,42 & 0,53 & 4,5 & $1,355 \pm 0,032$ & $>100$ & $0,139 \pm 0,007$ & $16 \pm 1$ \\
\hline okR7 & $7 \mathrm{c}$ & 1,32 & 0,49 & 13,1 & $1,444 \pm 0,016$ & $>1000$ & $0,200 \pm 0,006$ & $24 \pm 1$ \\
\hline okR10 & $7 \mathrm{c}$ & 1,61 & 0,43 & 17,0 & $1,398 \pm 0,016$ & $>1000$ & $0,471 \pm 0,011$ & $67 \pm 2$ \\
\hline ok101533 & 11 & 0,54 & 0,26 & 12,4 & $1,354 \pm 0,016$ & $>1000$ & $0,092 \pm 0,003$ & $11 \pm 1$ \\
\hline okR9 & 11 & 0,03 & 0,19 & 1,4 & $1,363 \pm 0,061$ & $>100$ & $0,402 \pm 0,019$ & $54 \pm 4$ \\
\hline ok101538d & 11 & 0,02 & 0,04 & 6,4 & $1,751 \pm 0,052$ & $>1000$ & $0,481 \pm 0,016$ & $68 \pm 3$ \\
\hline
\end{tabular}

Tableau 1 : Niveaux stratigraphiques, teneurs en Fe et Mn, teneurs en U, rapports d'activité et âges U-Th pour les échantillons osseux et de dentine de la grotte de Kůlna.

Table 1: Stratigraphical levels, $M n$ and Fe contents, U contents, activity ratios and U-Th ages for bone samples and dentine from Külna cave. 


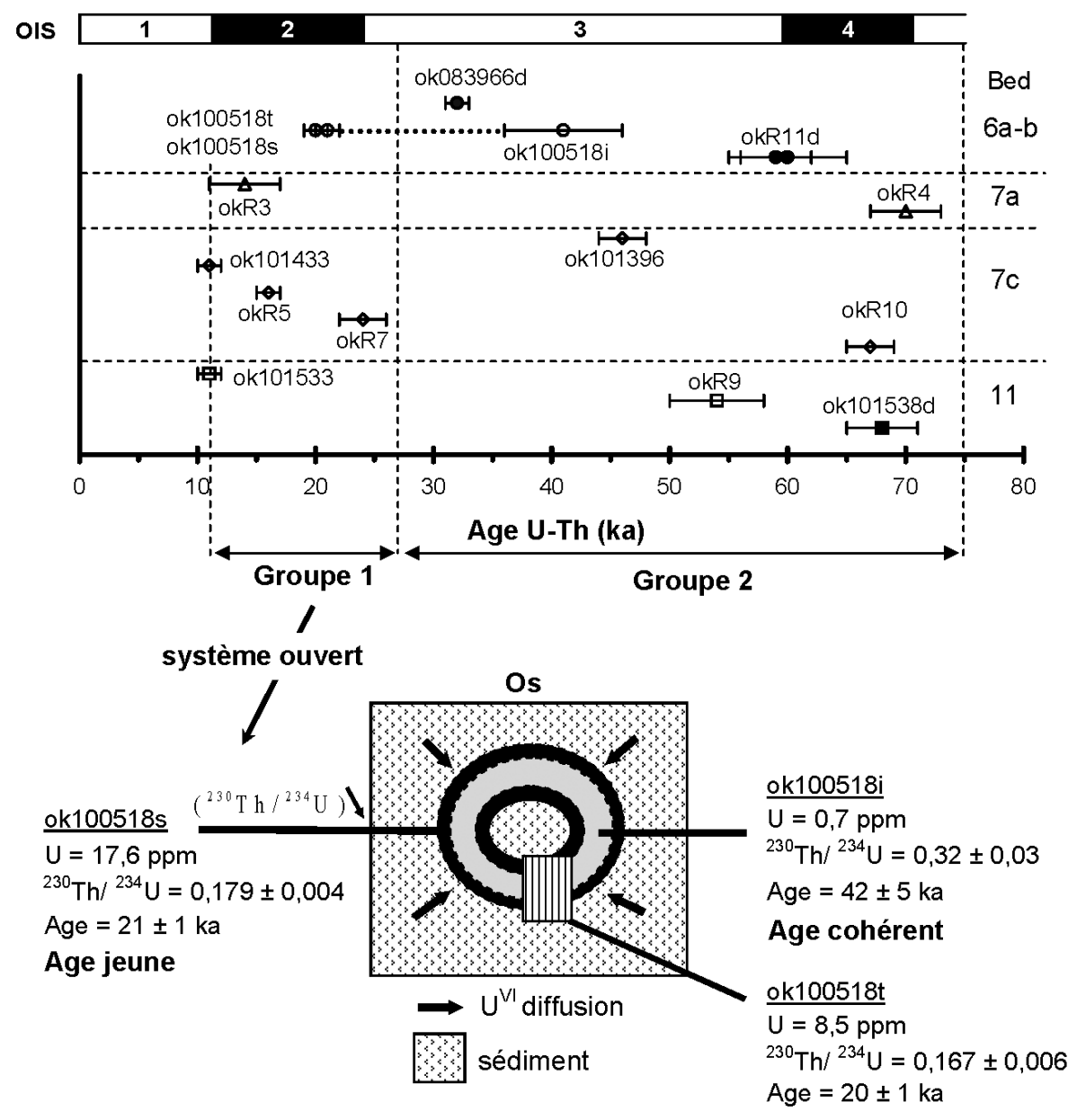

Age jeune

Figure 3 : Données U-Th des os de Kưlna. Les âges apparents sont présentés pour chaque niveau (6a-b, 7a, 7c, 11). Les datations de la surface, de l'intérieur et de l'os total (échantillon ok100518) sont détaillées.

Figure 3: U-Th data of Kulna bones. The apparent ages are presented for each level (6a-b, $7 a, 7 c, 11)$. The dates of the surface, the inner part and the whole bone (sample ok100518) are detailed.

sont plus cohérents avec les résultats de Rink et al. (1996) en dépit d'une dispersion significative des données. Ainsi, la formation des niveaux archéologiques semble correspondre aux stades isotopiques 3 et 4 . La présence de fer et de manganèse dans les os (Tableau 1) indique des changements redox qui ont sans doute eu pour conséquence des processus de migration d'uranium relativement complexes. Ils correspondent à des ouvertures du système se traduisant par une succession d'adsorption ou de perte d'uranium ayant une incidence sur la fiabilité des résultats de datation U-Th. Les données physico-chimiques ont souligné la complexité géochimique du site de Kůlna ne permettant pas cependant d'apporter de précision sur les âges des échantillons. Par ailleurs, il est préconisé par Shen (1996) de réaliser en parallèle à la méthode $\mathrm{U}-\mathrm{Th}$, une datation $\operatorname{par}^{231} \mathrm{~Pa} /{ }^{235} \mathrm{U}$ qui conduit à une comparaison avec une méthode de datation indépendante puisque la concordance éventuelle des deux méthodes (U-Th et U-Pa) démontre la fermeture du système et l'auteur souligne alors que l'âge est correct.

\section{Conclusion}

À Kůlna, la matière organique (collagène) et la phase minérale apatite des restes fauniques sont relativement bien préservées. Les analyses physico-chimiques montrent que la coloration plus ou moins importante des os de Kưlna est en grande partie liée à la présence d'oxydes de manganèse et 
de fer; des résultats qui ont également servi à une meilleure interprétation taphonomique des niveaux moustériens du site (Patou-Mathis et al., 2005). La méthode U-Th des restes osseux et dentaires n'a pas conduit à une datation précise des niveaux archéologiques en comparaison avec les autres méthodes. Les échanges entre les os et l'environnement sédimentaire mis en évidence par la présence significative du fer et du manganèse expliqueraient également des contaminations d'uranium par des migrations complexes entre l'encaissant et l'échantillon. Les âges U-Th sont dispersés entre les stades isotopiques 2,3 et 4 .

\section{Remerciements}

Cette étude a été financée par le programme CNRS «Paléoenvironnement, Evolution des Hominidés : Relations Hommes - Cultures - Environnements : le cas de l'Europe centrale au Paléolithique moyen" (dir. M. Patou-Mathis) et par le Cépam (dir. D. Binder). Les auteurs remercient P. Clément (Laboratoire de géologie, MNHN, Paris) pour les spectres de diffraction des RX et les deux rapporteurs.

\section{Bibliographie}

Bischoff, J. L., Rosenbauer, R., Tavoso, A. et Lumley De., H., 1988. A test of uranium-series dating of fossil tooth enamel: results from Tournal Cave, France, Applied Geochemistry, 3, p. $145-151$.

Bocherens, H., Tresset, A., Wiedemann, F., Giligny, F., Lafage, F., Lanchon, Y. et Mariotti, A., 1997. Diagenetic evolution of mammal bones in two French Neolithic sites, Bulletin de la Société géologique de France, 168, p. 555-564.

Ivanovich, M. et Harmon, R.S., 1992. Uranium-series Disequilibrium: Applications to Earth, Marine, and Environmental Sciences, Oxford, Clarendon Press, second edition, $910 \mathrm{p}$.

JeurneK, J., 1988. Anthropologishe funde aus der Kůlna-Hohle, in Die Erforschung Der Ki̊lna-Höhle 1961-1976, Brno, Morawske muezum, Anthropos Institut, p. 261-283.

Michel V., Falguères C. et Yokoyama Y., 1997. Teneur en uranium et datation U-Th des tissus osseux et dentaires fossiles de la grotte du Lazaret, Comptes Rendus de l'Académie des Sciences Paris, 325, p. 381-387.

Michel, V., Yokoyama, Y., Falguères, C. et Ivanovich, M., 2000. Problems encountered in the U-Th dating of fossil red deer jaws (bone, dentine, enamel) from Lazaret Cave: a comparative study with early chronological data, Journal of Archaeological Science, 27, p. 327-340.

Michel, V., Bocherens, H., Théry-Parisot, I., Valoch, K. et Valensi, P., 2006. Coloring and Preservation State of Faunal Remains from the Neanderthal Levels of Kůlna Cave (Czech Republic), Geoarchaeology: an International Journal, 5, p. 479501.

Моок, W. G., 1988. Radiocarbon-Daten aus der Kưlna-Höhle, In Die Erforschung Der Külna-Höhle 1961-1976, Brno, Morawske muezum, Anthropos Institut, p. 285.

Patou-Mathis, M., Auguste, P., Bocherens, H., Condemi, S., Michel, V., Moncel, M.-M., Neruda, P. et Valoch, K., 2005. Les occupations du Paléolithique Moyen de la grotte de Kůlna (Moravie, République Tchèque) : nouvelles approches, nouveaux résultats, in A. Tuffreau (éd.), Peuplements humains et variations environnementales au Quaternaire, Actes Colloque de Poitiers, 18-21 sept. 2000, Bar International Series, 1352, p. 69-94.

Pike, A. W. G., Hedges, R. E. M. et Van Calsteren, P., 2002. U-series dating of bone using the diffusion-adsorption model, Geochimica et Cosmochimica Acta, 66, p. 4273-4286.

Rae, A. M. et Ivanovich, M., 1986. Successful application of uranium series dating of fossil bone, Applied Geochemistry, 1, p. 419-426.

Rae, A. M., Hedges, R. E. M. et Ivanovich, M., 1989. Further studies for uranium-series dating of fossil bone, Applied Geochemistry, 4, p. 331-337.

Rink, W. J., Schwarcz, H., Valoch, K., Seitl, L. et Stringer, C. B., 1996. ESR dating of micoquian industry and Neanderthal remains at Kůlna Cave, Czech Republic, Journal of Archaeological Science, 23, p. 889-901.

SheN, G., 1996. U-series dating of fossil bones: results from Chinese sites and discussions on its reliability, Chinese Journal of Geochemistry, 15, p. 303-313.

Valoch, K., 1988. Die erforschung der Külna-Höhle 1961-1976, Brno, Morawske muezum, Anthropos Institut, 318 p. 